\title{
Mining Associated Ranking Patterns from Wireless Sensor Networks
}

\author{
Pu-Tai Yang
}

\begin{abstract}
Wireless Sensor Networks (WSNs) are complex networks consisting of many sensors which can detect and collect sensed data. Valuable information can be extracted from the sensed data of WSNs using data mining techniques, which provide unlimited possibilities of applications, and have been the object of an increasing number of studies in recent years. This study applies ranking sequence mining to WSNs. For example, if a sensor detects the following scenario during a time interval: $A$ occurs three times; $B$ occurs twice; $C$ occurs four times, where $A$, $B$ and $C$ are events. The information, $(C(4)>A(3)>B(2))$, can be abstractly expressed as a ranking sequence $(C \succ A \succ B)$, where " $\succ$ " indicates to the importance of the preceding item is greater than that of the succeeding item. To the best of our knowledge, there has been no research conducted on ranking pattern mining problems with WSNs. The objective of this study is to construct a novel model to discover interesting patterns from the sensed data of WSNs.
\end{abstract}

Index Terms-Data Mining, Wireless Sensor Networks, Ranking Sequences, Behavioral Patterns, Knowledge Discovery

\section{INTRODUCTION}

Wireless Sensor Networks (WSNs) have attracted considerable attention recently and have progressed tremendously because of their many potential applications [1]. A WSN consists of two types of communication nodes: sensors and base stations. The sensors selforganize and self-configure to establish a cooperative network; they can sense the environment, collect, receive, compress, relay and transmit a data stream to base stations. For example, a WSN used to monitor forest fires can raise an alarm in case of fire. More recently, interest in WSN research has shifted to reflect the current development of applications merging with other new technologies; one of them is data mining [2]. The sensors are not only able to detect and measure specific physical phenomena in the environment, but can also compute and compress information before or after the data stream transmission to the base station [2]-[4].

In WSNs, many large quantities of uncertain data are recorded. For example, sensors can continuously detect and collect environmental measurements such as humidity and temperature; however, the accuracy of the readings is dependent on the stability of the sensors or the noise of the environment. Other causes of uncertain data may include the incompleteness of input data. Under some strong assumptions, these sensed data can be transformed into a sequence. For example, $\left(s_{1}: A>B=C\right.$ ) is a temporal sequence, detected by the first sensor, which indicates that event $A$ happens earlier than event $B$, and event $B$ and $C$ happen at the same time. Another example is a ranking sequence, $\left(s_{1}: A \succ B \sim C\right)$, which indicates that sensor $s_{1}$ considers the importance of the event $A$ to be higher than that of event $B$, and that event $B$ is equally as important as to event $C$. Although the ranking importance is the product of subjective judgement, the ranking can be transferred by quantitative records under assumptions, e.g., their occurrence frequencies.

This study assumes the following scenario: sensors collect a lot of data, transform them into ranking sequences, and then transfer the ranking sequences back to the base station. The base station

P.-T. Yang is with the Department of Business Administration, Tunghai University, Taiwan

Corresponding author: Pu-Tai Yang, email: putai.yang@gmail.com can then execute a special data mining called "rank aggregation," which has been implemented in many data mining applications, such as decision support systems [5] and recommendation systems [6]-[8]. In rank aggregation, the base station (the decision-maker) discovers the consensus results from the sensed ranking sequence database; however, conflicts always exists because different sensors may provide different ranking sequences with diverse opinions. For example, given a group consisting of three sensors, they individually collect the occurrence frequencies of three events, $A, B$ and $C$ : (1) ( $s_{1}: A \succ B \succ C$ ), (2) ( $s_{2}: A \succ B \succ C$ ), and (3) (s $s_{3}: C \succ A \succ B$ ). All the sensors consider $A \succ B$, but the third sensor detects an abnormal event about the ranking of event $C$. In this example, the consensus pattern, $A \succ B$, is aggregated from the three sensors' ranking sequence and is logically persuasive. In the above situation, seeking consensus (compromised) results and solving discrepancies are necessary.

This study presents a conceptual framework for linking groupranking problems and WSNs. It also provides a corresponding algorithm which can discover interesting patterns across a ranking sequence database collected from a WSN. The research in [9]-[12] demonstrates that understanding which sensors detect these events is very useful. To the best of our knowledge, no research has attempted to integrate WSNs and ranking sequence database. This study thus proposes a novel concept: a sensor ranking database (SRD). How to discover the consensus ranking patterns and their associated sensors is an essential challenge in research and practice.

The remainder of this short paper is structured as follows: Section II reviews the data mining applications in WSNs and group-ranking problems. Section III describes the prpposed brand-new model concerning group-ranking problems in WSNs. An algorithm designed for this model, is then demonstrated and explained by a simple example in Section IV. The conclusions, discussions and our future work are discussed in Section V.

\section{RELATED WORK}

Generally speaking, the proposed model can be separated into two parts: the group-ranking problems, and data mining applied in WSNs. A brief review of the literature of the related group-ranking problems is given in Section II-A and data mining research for WSNs in Section II-B.

\section{A. Group Ranking Problems}

Association rules (also known as the market basket analysis problem) were first introduced in 1993 [13]. The initial purpose was used to analyze customer's shopping transactions in order to find frequent itemsets. Suppose a transaction database consists of many records, where each record contains a set of items. For example, if $50 \%$ of customers usually decide to buy beer, diapers and bread simultaneously, the itemset (beer, diaper, bread) is called a frequent itemset with a support of $50 \%$, where its support is over a user-specified minimum support threshold. The most important contribution in [13] is the Apriori principle, which is used to eliminate the number of the 
candidate itemsets: "If an itemset is frequent, then all of its supersets must be frequent." [14]

If the items are arranged or connected by relationship operators (e.g., temporal relationships or preference relationships), they constitute a sequence. A sequence with $k$ items is called a sequence with length $k$ (written as $k$-sequence). AprioriAll [15], the first algorithm for sequential pattern mining (SPM) problems, was proposed to discover frequent patterns in a sequence database. GSP [16], an Apriori-like algorithm, generalizes the SPM problem in [15] and improves the performance of AprioriAll. GSP, utilizing the Apriori principle and a generate-and-test strategy, can be simply summarized as two iterative phases: (1) candidate generation and (2) counting candidates. In GSP, $L_{k}$ is defined as a large set with length $k$, which consists of all frequent $k$-sequences. In the candidate generation phase, GSP joins two different sequences in $L_{k-1}$ and then generates a candidate $k$-sequence. In the second phase (counting candidates), GSP checks whether all candidate sequences are frequent sequences or not. All frequent $k$-sequences compose a new $L_{k}$ for the next computation until no further $L_{k}$ can be generated. The FP-growth algorithm [17] takes a totally different approach to mining frequent itemsets. The FP-growth algorithm does not use the generate-and-test strategy from GSP [16]; instead, it constructs a tree-based data structure (FP-tree) and discovers frequent itemsets directly from the tree. The FP-growth algorithm can be extended to sequential pattern mining problems [18].

When items are connected by preference operators $(\succ, \succeq, \sim, \prec$, $\preceq)$, they compose a ranking sequence. Generally speaking, the group ranking problems (rank aggregation) can be identified by the following three dimensions [19]:

1) The completeness of a ranking sequence: There are two types of ranking sequence: the total ranking approach [8], [20], [21], and the partial ranking approach [22]-[25]. For example, for a system with four items, $A, B, C$ and $D,(D \succ C \succ B \succ A)$ is a full ranking sequence because it contains all four items. On the contrary, $(D \succ B)$ is a partial ranking sequence because not all items appear in the sequence.

2) The input format of a ranking sequence: A group ranking problem is obtained by aggregating users' subjective thoughts. When users provide their preferences to the items, they can select (1) pairwise comparisons [8], (2) weight/scores [23], or (3) a ranking sequence directly [19], [26]. For $n$ items using pairwise comparisons, the relationships among them can be represented as an $n \times n$ matrix. For the weight/scores method, the user can assign scores to items via previously defined rules (e.g., 7-Likert scale). The first two formats can be derived to the ranking sequence format under some assumptions [5].

3) The outcome format of a ranking sequence: With different input format and algorithms, the outcome patterns also have two types: partial order and full order. Recently, [21] proposed a new type of outcome, preference graphs, to the SPM problems.

\section{B. Knowledge Discovery in WSNs}

Traditionally, knowledge discovery (data mining) in databases (KDD) [27] is a process to extract useful knowledge from a dataset or a database. Recently, KDD techniques have been extended to the WSNs field (called knowledge discovery in WSNs (KDW) [10]), and have attracted significant attention in many application areas [2], such as anomaly detection in railway data [28], relational temporal data mining [3], [29], [30] using Allen's temporal interval logic [31], object tracking [4], [11], [32], prediction of the location (target) of a missed reported event [33], and outlier (abnormal event) detection [34]. Until recently, on the whole there has been relatively little progress in the association rules applications to WSNs.
Boukerche and Samarah [9] proposed a comprehensive framework for sensor association rules. In their research, they are interested in whether sensors detect events, and not in the events' values, so the sensor pattern in [9] refers to $P=\left\{s_{1}, s_{2}, \ldots, s_{k}\right\} \subseteq S$, where $S$ is the set of sensors in a WSN. In their model, the time is divided into equal-sized time slots (epochs), and each epoch records the indices of event detecting sensors. Epochs consist of a sensor database. Compared to the traditional basket market analysis, epochs can be likened to the transactions in [35]. The sensor database can then be analyzed by the traditional data mining techniques (e.g., FP-growth tree [17] or SPADE [36]). Boukerche and Samarah also proposed a tree-based representation structure, the Positional Lexicographic Tree (PLT), to efficiently discover sensor behavioral patterns. Following the same framework, the researchers in [10] utilized the characteristics of WSNs and designed an in-network data reduction mechanism for reducing redundancies of sensed data. A relaxed version of sensor association rules concerning the coverage of sensors is also proposed in the same research. Recently, a mining algorithm, ASP [12], was proposed to consider the sensor data stream problem. In this problem, sliding observation windows generate different sensor datasets and the ASP algorithm captures the latest information for the current time.

In summary, there has been some research on the effects of sensor association rules; however, to the best of the authors' knowledge, there has been no research conducted on the connection of rank aggregation and WSNs. As stated in Section II-A, this study belongs to the following dimensions: ranking sequences, the partial input ranking approach, and the partial output ranking sequence. The main purpose of this study is to connect two different databases, the sensor database and the group-ranking sequence database, and to discover knowledge from them. Our proposed algorithm was developed and modified from the GSP algorithm [16], and is presented in the next section.

\section{Problem Definition}

As stated in the introduction, this study aims to discover sensor patterns from sensors' collected data; the details are stated as follows: A WSN can be modeled as a set of sensors, $\boldsymbol{S}=\left\{s_{1}, \ldots, s_{i}, \ldots, s_{|S|}\right\}$, where $s_{i}$ is the $i^{t h}$ sensor in the WSN and $i$ is the sensor index (the absolute value is used to represent the number of elements in a set; for instance, $|\boldsymbol{S}|$ denotes the number of sensors). This study considers a discrete-time system whose minimum time unit is round $t$, which allows the sensors to successfully complete data collection and necessary computations. Let $\boldsymbol{X}=\left\{x_{1}, x_{2}, \ldots, x_{|\boldsymbol{X}|}\right\}$ be the set of distinct pre-defined events. In the proposed model, sensors are not only able to detect events, but can also ranks those events into a sequence based on their occurrence frequencies or utility functions provided by users (see examples in Section I). At the end of the rounds, the sensors transmit the computed sequences back to the base station, which then discovers interesting patterns from the sequence database.

Next, the related terminology is defined with examples, and then the problem of mining sensor ranking patterns is defined.

Definition 1 (A ranking sequence). A ranking sequence is defined as $r s=\left(a_{1} \oplus_{1} \ldots \oplus_{j-1} a_{j} \oplus_{j} \ldots \oplus_{m-1} a_{m}\right)$, where $j$ is the event index in the ranking sequence; $a_{j} \in \boldsymbol{X}$; and $m=|r s|$ is the number of distinct events in the ranking sequence (also called the length of the ranking sequence). $\oplus_{j} \in\{\succ, \succeq, \sim\}$ refers to the ranking comparator between the two events $a_{j}$ and $a_{j+1}$. The ranking comparator " $\succ$ " denotes that the importance of the preceding events is greater than that of the succeeding event according to the user pre-defined regulations; " $\succeq$ " denotes that the importance of the preceding event is at least greater than that of the succeeding event; " " denotes that the importance is indifferent between the preceding and the succeeding events. Note 
that $\oplus \in\{\succ, \succeq, \sim\}$ (not including $\prec$ and $\preceq$ ) indicates that the events in a ranking sequence have been arranged in descending order. However, because of the numerous possible ordering due to the " " comparator, this study uses the idea of non-ambiguous sequence in [37] to ensure that the composition of the ranking sequence is unique. If the comparators among consecutive events are all " $\sim$ ", the events are arranged by lexicographic order.

Example 1. In this example, the occurrence frequencies are used to represent importance, whereas the measurements can be changed by the users. Suppose that a sensor detects the following events and their relative frequencies in a round: $A$ occurs four times; $B$ occurs five times; and $C$ occurs four times as $A$ does. The sensor then reports the following sensor ranking sequence back to the base station: $(B \succ$ $A \sim C)$. The ordering of $A$ and $C$ is decided by alphabetical order because $\oplus_{2}=$ " $\sim$ ".

Definition 2 (A relationship function). To a ranking sequence, $r s=\left(\ldots a_{j_{1}} \oplus_{j_{1}} \ldots \oplus_{t-1} a_{t} \oplus_{t} \ldots \oplus_{j_{2}-1} a_{j_{2}} \ldots\right)$, a relationship function $\operatorname{Rel}\left(r s, a_{j_{1}}, a_{j_{2}}\right)$ serves to obtain the relationship between the two events $\left\{a_{j_{1}}, a_{j_{2}}\right\}$ in a ranking sequence using the following definitions:

1) If $\forall \oplus_{t} \in\{\sim\}$, for $j_{1} \leq t \leq\left(j_{2}-1\right)$, then $\operatorname{Rel}\left(r s, a_{j_{1}}, a_{j_{2}}\right)$ is " $"$ ".

2) If $\exists \oplus t \in\{\succ\}$, for $j_{1} \leq t \leq\left(j_{2}-1\right)$, then $\operatorname{Rel}\left(r s, a_{j_{1}}, a_{j_{2}}\right)$ is " $\succ$ ".

3) Otherwise, $\operatorname{Rel}\left(r s, a_{j_{1}}, a_{j_{2}}\right)$ is “ $\succeq$ ".

Definition 3 (Ranking sequence containment). Consider two ranking sequences, $\alpha=\left(a_{1} \oplus_{1} \ldots \oplus_{j_{1}-1} a_{j_{1}} \oplus_{j_{1}} \ldots \oplus_{m_{1}-1} a_{m_{1}}\right)$ and $r s=$ $\left(b_{1} \oplus_{1} \ldots \oplus_{j_{2}-1} b_{j_{2}} \oplus_{j_{2}} \ldots \oplus_{m_{2}-1} b_{m_{2}}\right)$ where $m_{1} \leq m_{2} ; \forall a_{j_{1}}, b_{j_{2}} \in$ $\boldsymbol{X}$. It is claimed that $\alpha$ is contained in $r s$ (mathematically written as $\alpha \subseteq r s)$ if there are indices $1 \leq w_{1}<\ldots<w_{m_{1}} \leq m_{2}$ satisfy the following conditions:

1) $a_{1}=b_{w_{1}}, a_{2}=b_{w_{2}}, \ldots, a_{m_{1}}=b_{w_{m_{1}}}$;

2) $\operatorname{Rel}\left(\alpha, a_{j_{1}}, a_{j_{1}+1}\right)$ is contained in or equal to $\operatorname{Rel}\left(r s, b_{w_{j_{1}}}, b_{w_{j_{1}+1}}\right)$ for $j_{1}=1$ to $m_{1}-1$ ( $\succeq$ contains $\succ$ and $\sim$ ).

In summary, the elements (including events and comparators) of $\alpha$ are all in $r s$ and follow the same order and relationships.

Definition 4 (A sensor ranking database). A sensor ranking sequence, $s r s$, is a mixed data structure, $s r s_{i, t}=\left(s_{i}: r s\right)_{t}$, which refers to a ranking sequence, $r s$, detected and collected by the $i^{t h}$ sensor at round $t$. In this study, the base station records a sensor ranking database $(S R D)$, which consists of numerous sensor ranking sequences and their time delivered. For these ranking sequences collected in the same round, they are sorted by their sensor indexes. (See Figure 1 as an example.)

Definition 5 (Sensor ranking sequence containment). Given two sensor ranking sequences: $s r s_{i_{1}, t_{1}}=\left(s_{i_{1}}: \alpha_{1}\right)$, srs $s_{i_{2}, t_{2}}=\left(s_{i_{2}}, \alpha_{2}\right)$. The $s r s_{i_{1}, t_{1}}$ is contained in $s r s_{i_{2}, t_{2}}$ (written as $s r s_{i_{1}, t_{1}} \subseteq s r s_{i_{2}, t_{2}}$ if and only if $s_{i_{1}}=s_{i_{2}}$ and $\alpha_{1} \subseteq \alpha_{2}$.

Example 2. Consider the $S R D$ in Table 1, it is claimed that the srs $_{1,1}=(A \succeq B \succ C \sim D)$ contains the $\operatorname{sr} s_{1,3}=(A \succ B)$ because the following conditions are satisfied:

1) These two ranking sequences are provided by the same sensor, $s_{1}$.

2) $(B \succ D)$ is contained in $(A \succeq B \succ C \sim D)$.

http://dx.doi.org/10.15242/IIE.E0116025

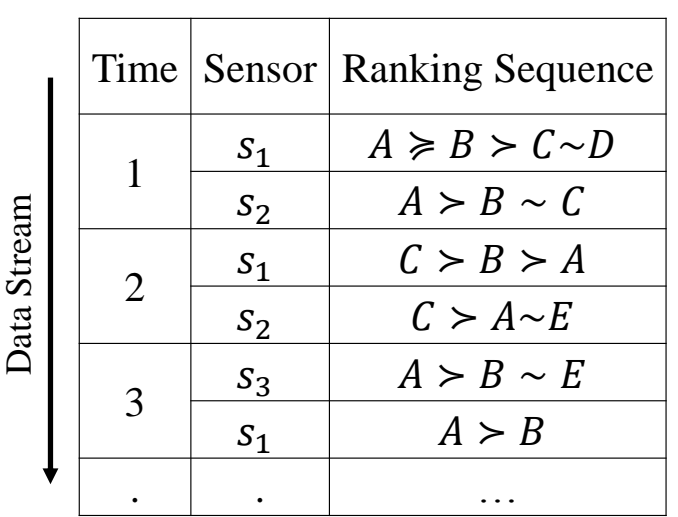

Fig. 1: Example illustrating a sensor ranking database from a WSN' data stream.

Definition 6 (Supports). The sensor ranking support (srsup) of a sensor ranking sequence, $\gamma$, to a given $S R D$ is defined as follows:

$$
\begin{aligned}
\operatorname{srsup}(\gamma, S R D) & =\frac{\sigma(\gamma, S R D)}{|S R D|} \\
& =\frac{\left|\left\{s r s_{i, t} \mid\left(\gamma \subseteq \operatorname{srs}_{i, t}\right) \cap\left(\operatorname{srs}_{i, t} \in S R D\right)\right\}\right|}{|S R D|},
\end{aligned}
$$

As a result, srsup is a real number between 0 and 1 .

Definition 7 (Frequent patterns and large sets). A sensor ranking pattern is defined as "frequent" if its support is greater than or equal to a minimum user-specified threshold, min_sup. A set of frequent (sensor ranking) patterns with the same length $m$ is defined as a large set, $\boldsymbol{L}_{m}$.

The problem in this study can be described as follows: Given an $S R D$, and a user-specified support threshold, min_sup, the task is to discover all frequent sensor ranking patterns.

\section{The Proposed Algorithm}

In this section, we introduces the proposed algorithm, Mining Associated Rankings from Sensors (MARS), to discover the sensor ranking patterns from an $S R D$. The development of the MARS algorithm is based on GSP [16]. In explaining the MARS algorithm, this paper uses the $S R D$ in Figure 1 as an auxiliary example. In the example, min_sup $=0.3$ is used, which also means that the minimum support count is min_sup $\times|S R D|=0.3 \times 6=1.8$.

First, the MARS algorithm scans the $S R D$ (Line 3) to obtain all frequent srs with only one single event. The MARS algorithm then generates the first large set with length one, $\boldsymbol{L}_{1}$, by comparing their supports to the minimum support thresholds:

$$
\begin{aligned}
& \boldsymbol{L}_{1}= \\
& \quad\left\{\text { srs }=\left(s_{i}: x\right) \mid(x \in \boldsymbol{X}) \cap(\sigma(x) \geq|S R D| \times \text { min_sup })\right\} .
\end{aligned}
$$

See Table I as an example. Only those sensor ranking sequences whose support counts are greater than or equal to 2 are collected into $\boldsymbol{L}_{1}$. For example, $\left(s_{1}: D\right),\left(s_{2}: B\right),\left(s_{2}: E\right),\left(s_{3}: A\right),\left(s_{3}: B\right)$, and $\left(s_{3}: E\right)$ are not collected in $\boldsymbol{L}_{1}$.

In the second step (Lines 7-10), the Apriori principle [15] and $L_{m-1} \times L_{m-1}$ method [14] are used to reduce the time complexity of the MARS algorithm. The $L_{m-1} \times L_{m-1}$ method generates candidates sensor ranking sequences using merging a pair of frequent $(m-1)$ sensor ranking patterns only if they share the same sensor index and the same $(m-2)$ itemset. As a result, for the sub-dataset collected from different sensors, it is possible to separately process them to 


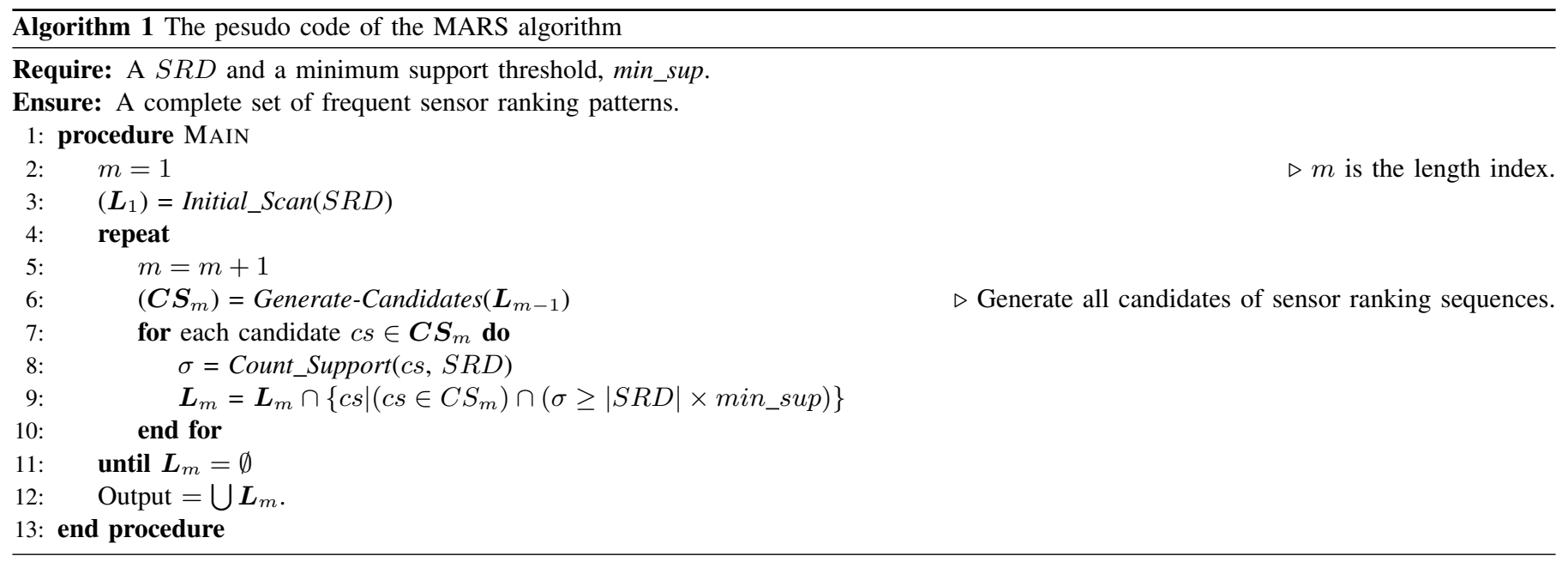

TABLE I: An example illustrating $\boldsymbol{L}_{1}$.

\begin{tabular}{|c|c|c|c|c|c|}
\hline$s r s$ & $\sigma$ & $s r s$ & $\sigma$ & $s r s$ & $\sigma$ \\
\hline$\left(s_{1}: A\right)$ & 3 & $\left(s_{2}: A\right)$ & 2 & $\left(s_{3}: A\right)$ & 1 \\
\hline$\left(s_{1}: B\right)$ & 3 & $\left(s_{2}: B\right)$ & 1 & $\left(s_{3}: B\right)$ & 1 \\
\hline$\left(s_{1}: C\right)$ & 2 & $\left(s_{2}: C\right)$ & 2 & $\left(s_{3}: E\right)$ & 1 \\
\hline$\left(s_{1}: D\right)$ & 1 & $\left(s_{2}: E\right)$ & 1 & & \\
\hline
\end{tabular}

reduce the number of comparisons. For example, it is unnecessary to scan $s_{3}$ 's sensor ranking sequences because none of them are frequent. For $s_{2}$ 's $S R D$, the only five candidates generated from events $A$ and $C$ are $(A \succ C),(A \succeq C),(A \sim C),(C \succ A)$, and $(C \succeq A)$. None of these are frequent. For $s_{1}$ 's $S R D$, there are 15 candidate ranking sequences; only $(A \succ B)$ and $(B \succ C)$ are frequent. In summary, only two sensor ranking sequences, $\left(s_{1}: A \succ B\right)$ and $\left(s_{2}: B \succ C\right)$ are frequent sensor ranking patterns in the $S R D$ in Figure 1.

\section{Discussions ANd Future Work}

This short report describes a novel model for combining ranking sequence mining and WSNs. It also proposes a related algorithm, MARS, to be applied with the above model. Although the proposed MARS algorithm is designed to discover all sensor ranking patterns, other possibilities were discovered. Future work will first compare the proposed algorithm by implementing it on a WSN simulator to examine its performance. Second, the effectiveness of the proposed algorithm will be explored under a greater variety of situations. Simulations are underway to demonstrate various trade-offs among different scenarios in order to validate the effectiveness of the proposed algorithm.

\section{ACKNOWLEDGEMENT}

The authors would like to thank the Ministry of Science and Technology, Taiwan for financially supporting this research under MOST 103-2221-E-029-019-.

\section{REFERENCES}

[1] L. Borges, F. Velez, A. Lebres, Survey on the characterization and classification of wireless sensor network applications, IEEE Communications Surveys \& Tutorials 16 (4) (2014) 1860-1890.

[2] A. Mahmood, K. Shi, S. Khatoon, M. Xiao, Data mining techniques for wireless sensor networks: A survey, International Journal of Distributed Sensor Networks 2013. doi:doi:10.1155/2013/406316.

[3] V. Jakkula, D. J. Cook, Mining sensor data in smart environment for temporal activity prediction, in: Poster session at the ACM SIGKDD, 2007.
[4] V. S. Tseng, E. H.-C. Lu, Energy-efficient real-time object tracking in multi-level sensor networks by mining and predicting movement patterns, Journal of Systems and Software 82 (4) (2009) 697-706.

[5] Y. Chen, L. Cheng, W. Hsu, A new approach to the group ranking problem: Finding consensus ordered segments from users preference data, Decision Sciences 44 (2013) 1091-1119.

[6] Q. Li, S. H. Myaeng, B. M. Kim, A probabilistic music recommender considering user opinions and audio features, Information Processing \& Management 43 (2007) 473-487.

[7] R. K. Pon, A. F. Crdenas, D. Buttler, T. J. Critchlow, Measuring the interestingness of articles in a limited user environment, Information Processing \& Management 47 (2011) 97-116.

[8] S.-M. Chen, S.-H. Cheng, T.-E. Lin, Group decision making systems using group recommendations based on interval fuzzy preference relations and consistency matrices, Information Sciences 298 (2015) 555 - 567.

[9] A. Boukerche, S. Samarah, A novel algorithm for mining association rules in wireless ad hoc sensor networks, IEEE Transactions on Parallel and Distributed Systems 19 (7) (2008) 865-877.

[10] A. Boukerche, S. Samarah, In-network data reduction and coverage-based mechanisms for generating association rules in wireless sensor networks, IEEE Transactions on Vehicular Technology 58 (8) (2009) 4426-4438.

[11] S. Samarah, M. Al-Hajri, A. Boukerche, A predictive energy-efficient technique to support object-tracking sensor networks, IEEE Transactions on Vehicular Technology 60 (2) (2011) 656-663.

[12] M. M. Rashid, I. Gondal, J. Kamruzzaman, Mining associated patterns from wireless sensor networks, IEEE Transactions on Computers 64 (7) (2015) 1998-2011.

[13] R. Agrawal, T. Imieliński, A. Swami, Mining association rules between sets of items in large databases, in: Proceedings of the 1993 ACM SIGMOD International Conference on Management of Data, ACM, NY, USA, 1993, pp. 207-216.

[14] P.-N. Tan, M. Steinbach, V. Kumar, Introduction to Data Mining, Addison-Wesley, 2014.

[15] R. Agrawal, R. Srikant, Mining sequential patterns, in: Proceedings of the Eleventh International Conference on Data Engineering, IEEE, 1995, pp. 3-14.

[16] R. Srikant, R. Agrawal, Mining sequential patterns: Generalizations and performance improvements, in: Proceedings of the 5th International Conference on Extending Database Technology: Advances in Database Technology, Springer-Verlag, London, UK, 1996, pp. 3-17.

[17] J. Han, J. Pei, Y. Yin, Mining frequent patterns without candidate generation, in: ACM SIGMOD Record, Vol. 29, ACM, 2000, pp. 1-12.

[18] J. Pei, J. Han, B. Mortazavi-Asl, H. Pinto, Q. Chen, U. Dayal, M.-C. Hsu, Prefixspan: mining sequential patterns efficiently by prefix-projected 
pattern growth, in: Proceedings of the 17th International Conference on Data Engineering, 2001, pp. 215-224.

[19] Y.-L. Chen, L.-C. Cheng, Mining maximum consensus sequences from group ranking data, European Journal of Operational Research 198 (2009) 241-251.

[20] O. S. Vaidya, S. Kumar, Analytic hierarchy process: An overview of applications, European Journal of Operational Research 169 (1) (2006) $1-29$.

[21] Y.-L. Chen, L.-C. Cheng, P.-H. Huang, Mining consensus preference graphs from users' ranking data, Decision Support Systems 54 (2013) $1055-1064$.

[22] S. Alonso, E. Herrera-Viedma, F. Chiclana, F. Herrera, A web based consensus support system for group decision making problems and incomplete preferences, Information Sciences 180 (23) (2010) 4477 4495.

[23] W. D. Cook, B. Golany, M. Kress, M. Penn, T. Raviv, Optimal allocation of proposals to reviewers to facilitate effective ranking, Management Science 51 (2005) 655-661.

[24] D. S. Hochbaum, A. Levin, Methodologies and algorithms for grouprankings decision, Management Science 52 (2006) 1394-1408.

[25] W. D. Cook, B. Golany, M. Penn, T. Raviv, Creating a consensus ranking of proposals from reviewers partial ordinal rankings, Computers \& Operations Research 34 (2007) 954-965.

[26] Y.-L. Chen, L.-C. Cheng, An approach to group ranking decisions in a dynamic environment, Decision support systems 48 (2010) 622-634.

[27] C.-K. Huang, P.-T. Yang, K.-Y. Hsieh, Knowledge discovery of consensus interval-based temporal patterns in group decision-making, under revision (2015).

[28] J. Rabatel, S. Bringay, P. Poncelet, SO_MAD: SensOr Mining for Anomaly Detection in railway data, in: Advances in Data Mining. Applications and Theoretical Aspects, Springer, 2009, pp. 191-205.

[29] F. Esposito, T. M. Basile, N. Di Mauro, S. Ferilli, A relational approach to sensor network data mining, in: Information Retrieval and Mining in Distributed Environments, Vol. 324 of Studies in Computational Intelligence, Springer, 2011, pp. 163-181.

[30] T. M. Basile, N. Di Mauro, S. Ferilli, F. Esposito, Relational temporal data mining for wireless sensor networks, in: AI*IA 2009: Emergent Perspectives in Artificial Intelligence, Springer, 2009, pp. 416-425.

[31] J. F. Allen, Maintaining knowledge about temporal intervals, Communications of the ACM 26 (1983) 832-843.

[32] K. W. Lin, M.-H. Hsieh, V. S. Tseng, A novel prediction-based strategy for object tracking in sensor networks by mining seamless temporal movement patterns, Expert Systems with Applications 37 (4) (2010) 2799 $-2807$.

[33] S. Samarah, A. Boukerche, A. Habyalimana, Target association rules: A new behavioral patterns for point of coverage wireless sensor networks, IEEE Transactions on Computers 60 (6) (2011) 879-889.

[34] Y. Zhang, N. Meratnia, P. Havinga, Outlier detection techniques for wireless sensor networks: A survey, IEEE Communications Surveys \& Tutorials 12 (2) (2010) 159-170.

[35] R. Agrawal, R. Srikant, Fast algorithms for mining association rules, in: Proc. 20th int. conf. very large data bases, VLDB, Vol. 1215, 1994, pp. 487-499.

[36] M. J. Zaki, Spade: An efficient algorithm for mining frequent sequences, Machine Learning 42 (1-2) (2001) 31-60.

[37] S.-Y. Wu, Y.-L. Chen, Mining nonambiguous temporal patterns for interval-based events, IEEE Transactions on Knowledge and Data Engineering 19 (2007) 742-758. 